Revue d'histoire de l'Amérique française

REVUE D.HISTOIRE DE L'AMÉRIQUE FRANÇAISE

\title{
Quelques aspects de la dissolution de la Compagnie de M. de Monts, 1607
}

\section{Th. J. Kupp}

Volume 24, numéro 3, décembre 1970

URI : https://id.erudit.org/iderudit/302988ar

DOI : https://doi.org/10.7202/302988ar

Aller au sommaire du numéro

Éditeur(s)

Institut d'histoire de l'Amérique française

ISSN

0035-2357 (imprimé)

1492-1383 (numérique)

Découvrir la revue

Citer cet article

Kupp, T. J. (1970). Quelques aspects de la dissolution de la Compagnie de M. de Monts, 1607. Revue d'histoire de l'Amérique française, 24(3), 357-374.

https://doi.org/10.7202/302988ar d'utilisation que vous pouvez consulter en ligne.

https://apropos.erudit.org/fr/usagers/politique-dutilisation/ 


\title{
QUELQUES ASPECTS DE LA DISSOLUTION DE LA COMPAGNIE DE M. DE MONTS, 1607
}

Brève étude de l'influence du commerce hollandais sur les premiers efforts concertés au dix-septième siècle en vue de coloniser le Canada.

\author{
TH J. Kupp \\ Université de Victoria, C.B.
}

Celui qui étudie l'histoire du Canada n'ignore pas la "menace venant du sud", s'entend la menace que constitue pour l'existence du Canada comme nation la situation climatique, économique et politique qui prévaut au sud de ses frontières. Ce qu'on sait moins, c'est que cette menace remonte à l'époque où la France commença à s'attaquer résolument à la colonisation du Canada. Lescarbot, Champlain et les Jésuites affirment que les trafiquants hollandais étaient résolus à s'emparer du commerce des fourrures de la colonie laurentienne. Apparemment, ces marchands hollandais, qu'on n'avait pas de peine à retrouver dans tous les carrefours commerciaux du monde au tournant du seizième siècle, étaient devenus conscients des conditions favorables que présentait le commerce canadien et de l'excellence de ses fourrures. Tout comme les Français et les Anglais auparavant, c'est par les pêcheries de Terre-Neuve que les marchands hollandais en vinrent à connaître les fourrures d'Amérique. En 1590, des marchands d'Amsterdam envoyèrent un des leurs, Jacques de la Faille, faire un voyage de reconnaissance en Méditerranée en vue d'un rapport sur les possibilités commerciales du Levant. Il rapporta que dans le sud de l'Italie, en Grèce et dans la Crète, il y avait une forte demande pour le lin, le saumon et l'anguille salés, le hareng séché, le poil servant au blanchissage de maisons à la chaux, et le poisson de Terreneuf séché au vent. C'est là l'origine des échanges des Hollandais avec le Levant qui constituèrent un secteur important de leur empire commercial jusqu'à bien avant dans le dixhuitième siècle ${ }^{1}$. De 1592 à 1602 , on entreprit nombre de voyages pour se procurer la morue de Terre-Neuve dont on avait besoin, d'abord à Dartmouth et à Plymouth, et après 1597 directement aux Grands Bancs, où on l'acheta des pêcheurs français et anglais. ${ }^{2}$ A lire certains contrats, il semble qu'après 1597 on trans-

${ }^{1}$ Archives de la ville d'Amsterdam, collection van der Meulen, no 118. Désormais AVA.

2 On peut trouver une liste complète de tous les contrats disponibles touchant ces voyages à Terre-Neuve, aux AVA, collection J. W. Ijzerman,

[ 357 ]

RHAF, vol. 24, no 3 (décembre 1970) 
porta de Hollande ou d'Angleterre à Terre-Neuve certaines marchandises qui auraient pu servir pour le troc avec les Indiens. Il n'est pas absolument certain que ces Hollandais aient pris une part active dans le commerce des fourrures sur les côtes du Canada, mais il va sans dire qu'ils ne pouvaient pas ne pas être informés par d'autres du caractère lucratif du commerce des fourrures avec les indigènes. Il est donc remarquable que l'attention des Néerlandais ne se soit pas immédiatement tournée vers l'Amérique du Nord. De fait, les voyages à TerreNeuve cessèrent presque complètement après 1602 et ne reprirent qu'au début des années 1620. Il est très probable que l'intérêt du commerce hollandais se soit tourné ailleurs par suite de la fondation, en 1602, de la Compagnie hollandaise des Indes orientales, dans laquelle investirent gros les premiers qui avaient participé au commerce entre la Hollande et TerreNeuve, comme Isaac le Maire. C'est aussi à cette époque que devait se décider la grande lutte entre les commerçants hollandais et ceux de l'English Muscovy Company pour le monopole du commerce russe des fourrures. ${ }^{3}$ Mais en 1606, les marchands de la république hollandaise tournèrent soudain leur attention vers le commerce canadien des fourrures d'une très singulière façon. Cette année-là, un groupe de marchands d'Amsterdam attaqua la Compagnie française de Monts et lui fit encourir des pertes si lourdes qu'elle dut être dissoute. Pourquoi soudainement, semble-t-il, nombre de commerçants hollandais en vinrent-ils à s'intéresser aux fourrures canadiennes ? La réponse à cette question viendra peut-être d'une étude attentive des événements qui ont amené la dissolution de la Compagnie de Monts en 1607:

... que la société estoit rompuë, d'autant que contre l'Edit du Roy les Holandois, conduits par un traitre François nommé La Ieunesse, avoient l'an precedent enlevé les Castors

contrats d'affrètement d'Amsterdam (Espagne et Portugal), 1592-1602, Economisch Historisch Jaarboek (La Haye, 1931), 163, 291. Aussi AVA, Archives notariales, contrats Wiltbraet-'t Hart (1601); Lubner-Geusenbroeck (1596).

3 Pour les relations commerciales Hollande-Russie de cette période on peut consulter D. S. van Zuiden, "Contributions aux connaissances concernant le commerce Hollande-Russie". Publications de documents originaux des Archives notariales d'Amsterdam, Economisch Historisch Jaarboek (La Haye, 1916). Pour les sources russes: Inna Lubimenko, Les Relations commerciales et politiques de l'Angleterre avec la Russie avant Pierre le Grand (Paris, 1933); "The Struggle of the Dutch with the English for the Russian market in the seventeenth century", Transactions of the Royal Historical Society, $4^{\mathrm{e}}$ r., vol. 7 (1924); T. S. Willan, The early History of the Russia Company 1553-1603 (Manchester, 1956). 
et autres pelleteries de la grande Riviere de Canada, chose qui tournoit au grand detriment de la société, laquelle partant ne pouvoit plus fournir aux frais de l'habitation de delà, comme elle avoit fait par le passé... Or cette envie sur le traffic des Castors avec les Sauvages ne s'est pas seulement glissée és coeurs des Holandois, mais aussi des marchans François, de maniere qu'en fin le privilege qui avoit esté baillé audit sieur de Monts pour dix ans a esté revoqué. ${ }^{4}$

Voilà la notice nécrologique consacrée à la Compagnie de Monts par Lescarbot. Elle constitue une mise en accusation de ceux qui ont amené la fin d'une entreprise capable d'asseoir solidement le développement de la Nouvelle-France. On a fait peu de cas de ce passage. Il est cependant important, puisque la fin de la Compagnie de Monts signifiait le début d'une vigoureuse concurrence hollandaise pour les fourrures du Canada, concurrence qui allait durer jusqu'au dix-huitième siècle et affecter durement la Nouvelle-France. ${ }^{5}$ Les archives notariales de la Ville d'Amsterdam jettent quelque lumière sur les événements de 1606.

$\mathrm{Au}$ printemps de 1606, quelques-uns des principaux marchands d'Amsterdam avaient formé une compagnie dont un des buts essentiels était le commerce des fourrures du Canada et la course sur les territoires de pêche de Terre-Neuve ou dans les environs. Il étaient huit en tout à participer à l'entreprise. C'étaient le capitaine Hendrick Lonck et les marchands Bernard Berrewijns, Hans Hunger, Jorgen Timmermans, Hans Franck, Jan Cupper, Hannes Heusen et Louis Delbecque. Cette compagnie que nous appellerions la Compagnie Berrewijns, affréta un vaisseau de 320 tonnes, le Lion Blanc, propriété des actionnaires, et l'équipa pour parer à toute éventualité. Le capitaine était Hendrick Lonck qui fut par la suite amiral de la flotte de la Compagnie des Indes occidentales; le second était Hendrick Cornelisz, et le chirurgien Geraert Claessen. Tous les participants étaient des citoyens d'Amsterdam. ${ }^{6}$ En juillet ou

${ }^{4}$ Lescarbot, History of New France, par W. L. Grant, éditeur (Toronto, 1911), II : 350-352; texte français dans: Marc Lescarbot, Histoire de la Nouvelle France contenant les navigations, découvertes et habitations faites par les Francois ès Indes occidentales et Nouvelle France avec les muses de la Nouvelle France (3 vol., Paris, Tross, 1866), II: 562-565.

5 Th. J. Kupp, The Fur Trade Relations New Netherland-New France, 1600-1664. Thèse de doctorat non publiée, Université du Manitoba (1968).

6 AVA, Archives notariales, no 106, f. 54v-55; no 105, f. 198-199. Archives des Etats généraux, La Haye, Archives de l'Amirauté, no 1359. Charles Beaurepaire, "La Compagnie de Monts", La Normandie, 9e année, no 1 ( $2^{\mathrm{e}}$ série, Rouen 1893 ). 
août 1606, Lonck rencontra à Tadoussac deux des quatre navires dont se composait la flotte de la Compagnie de Monts. L'un se tenait à Port-Royal, un autre protégeait la côte des intrus, et deux trafiquaient le long de la côte. On peut conjecturer ce qui arriva. Il est probable que les Hollandais tentèrent de trafiquer avec les indigènes et furent surpris par les deux navires français. Ces derniers n'étaient pas de taille à se mesurer au vaisseau hollandais fortement armé et leurs fourrures furent pillées. Pour prévenir toute nouvelle ingérence française dans leur trafic, les Hollandais enlevèrent des navires de Monts les canons, les montures et les munitions, et continuèrent à troquer avec les Indiens. En partant de Tadoussac, les Hollandais découvrirent dans la baie SainteMarie à Terre-Neuve deux navires, un espagnol et l'autre portugais, qu'ils soulagèrent de leurs cargaisons de baleine, de morue et autres marchandises. Les navires furent considérés comme des prises, l'Espagne et le Portugal étant en guerre avec la république de Hollande. On n'avait pas enlevé ses navires à de Monts, parce que la France était une alliée de la Hollande, et plus probablement parce que durant les quelques dernières années l'ambassadeur de la France à La Haye, Buzanval, avait été inondé de plaintes au sujet de corsaires hollandais. On ne dit pas s'il était présent lors du vol de ses vaisseaux. C'est probablement Jean Ott, un des capitaines de la compagnie française qui commandait - du moins il est fait mention de lui. ${ }^{7}$ La Compagnie de Monts avait dû faire une perte importante, puisque dès son retour en France, de Monts se plaignit à la Cour. Le roi Henri IV regarda l'action des Hollandais comme une menace directe à sa politique économique, et par l'intermédiaire de son ambassadeur, logea une plainte énergique auprès des Etats généraux de Hollande. ${ }^{8}$

Ce qu'il y a d'intéressant dans cette attaque hollandaise contre le commerce des fourrures de la Nouvelle-France, c'est que le subrécargue du Lion Blanc était le trafiquant rouennais Nicolas de Bancquemaire. ${ }^{9}$ C'est probablement lui le traître "La Jeunesse", mentionné par Lescarbot. Il se peut que ce soit là un sobriquet pour distinguer le jeune Nicolas du vieux Nicolas

7 AVA, Archives notariales, no 195 , f. $85 \mathrm{v}-86 \mathrm{v}$; no 106 , f. $54 \mathrm{v}-55$. Archives nationales de France, Registre de Cuvillier, XV, 19, f. 945. Archives de la Seine Maritime, Tabellionage de Rouen (meubles), $1^{\text {ère }}$ série, $1^{\text {er }}$ janvier-30 juin 1607. Archives des Etats Généraux, La Haye, Résolutions des Etats Généraux du 17 mars 1601 et du 8 octobre 1605 .

$8 \mathrm{M}$. Berger de Xivrey, éd., Recueil des Lettres missives de Henri IV (Paris, 1858), VII: 465-466.

9 AVA, Archives notariales, no 106, f. 54v-55. 
de Bancquemaire qui, en 1576, avait été lui-même victime des corsaires hollandais. ${ }^{10}$ On peut tout au plus supposer comment de Bancquemaire en vint à être impliqué dans l'entreprise des Hollandais. Il était probablement du nombre des marchands exclus du monopole établi par de Monts. Les Bancquemaire avait des relations d'affaires avec la firme hollandaise des van Liebergen, commerçants de fourrures établis à Rouen et à Amsterdam. Il semblerait que les Bancquemaire ont pris part à des transactions commerciales louches, qui les ont souvent amenés devant les tribunaux de France et de Hollande. En 1611, Arnout Van Liebergen fit arrêter Nicolas de Bancquemaire pour avoir fait tort à la réputation de son père, Jan van Liebergen et à la sienne. Cette même année, Jacques de Bancquemaire fit une réclamation contre Balthasar de Visschere (Pescator), beau-frère d'Arnout van Liebergen. Les de Visschere commerçaient avec Nathaniel de Bancquemaire. ${ }^{11}$ De Monts fut impliqué dans un procès avec Nicolas de Bancquemaire qui dura plus de dix ans..$^{12}$ Les événements qui suivirent le raid des Hollandais contre la Compagnie de Monts en 1606 sont pour beaucoup dans ces fâcheux rapports.

En janvier 1607, Henri IV écrivit aux Etats généraux de Hollande pour se plaindre de l'attaque hollandaise contre une compagnie opérant en vertu d'une charte royale sur des territoires revendiqués par le roi de France. ${ }^{13}$ On s'empressa de s'occuper de sa plainte, car la France aidait la république de Hollande dans la lutte qu'elle menait contre l'Espagne pour son indépendance, en lui fournissant des secours financiers consi-

10 Décision de la "Prize Court of Zeeland", no CCXX, 1 $1^{\text {er }}$ nov. 1576. Dans Bijdragen en Mededeelingen van het Historisch Genootschap (Amsterdam, 1916), vol. 37.

11 Arnout van Liebergen était un commerçant de fourrures d'Amsterdam et fut un des premiers à entreprendre la traite des fourrures dans la région de la rivière Hudson. On trouvera des renseignements sur la branche hollandaise de la famille dans S. Hart, The Prehistory of the New Netherland Company (Amsterdam, 1959), 58. Jan van Liebergen demeurait à Rouen et était lié aux commercants de fourrures et aux fabricants de chapeaux. Son fils, Jan, épousa Suzanne Balcot en 1616; sa fille Marie a épousé Louis Legrand en 1614. L'autre fille, Anne, s'est mariée deux fois: d'abord à Jean Clément de Quevilly, de Rouen, et en 1640, à David Des Essarts, un médecin. On trouvera d'autres renseignements sur la branche française des van Liebergen, dans le Bulletin de la Commission des Eglises wallonnes (La Haye, 1892, 1896), $2^{\text {e }}$ série, I: 129-151 et V: 203-224.

12 Bibliothèque Nationale de France, Interrogatoire de Pierre de Gua, sieur de Monts, 8, $8^{\circ} \mathrm{Z} 11535$. - Archives nationales de France, V6, 1211, pièce 9, f. 33 : V5, 1216, pièce $24, f$. 526 .

13 Berger de Xivrey, éd., Lettres missives de Henıi IV, VII : 465-466. 
dérables. Le 2 février 1607, aux Etats généraux des sept Provinces Unies, on lut une lettre dans laquelle Sa Majesté se plaignait qu'un navire appelé le Lion Blanc, équipé pour la guerre dans la ville d'Amsterdam, était entré dans les eaux canadiennes et avait abordé en Nouvelle-France, un pays que le roi de France avait découvert et commencé à peupler il y avait plus de cent ans. Le Lion Blanc avait dépouillé deux vaisseaux appartenant à un gentilhomme de Sa Majesté, M. de Monts, de leurs canons, montures et munitions et s'était emparé des fourrures des indigènes. Le roi exigeait une restitution et demandait aux Etats généraux de défendre à leurs marchands d'entrer au Canada et de faire le commerce dans ce pays. Les Etats généraux envoyèrent la lettre à l'Amirauté d'Amsterdam, en lui demandant d'aviser et d'agir par rapport aux canons et à l'équipement volé. L'Amirauté répondit, le 19 février 1607, que les canons, montures et munitions avaient été confisqués et seraient retournés à de Monts lorsque reçus. La ville d'Amsterdam ne consentait pas pour autant à abandonner la liberté des mers par égard pour le roi de France. Les Etats généraux partagèrent cette attitude. Indiquant que ce serait interdire aux citoyens hollandais la navigation vers le Canada, le gouvernement hollandais déclara dans sa réponse qu'il espérait que ce ne fut pas l'intention de Sa Majesté de gêner la liberté commerciale de la Hollande. ${ }^{14}$ Cependant, l'affaire de Monts n'en resta pas là.

De Monts espérait probablement avoir gain de cause contre la Compagnie hollandaise, avec l'aide du roi son maître. Il dépêcha à La Haye son secrétaire Jean Ralluau, avocat. Chose curieuse, ce dernier fit le voyage de Hollande sur un des bateaux de la compagnie Berrewijns. Il était accompagné d'un "nègre", Mathijs (Mathieu) Da Costa. Le compagnon de Ralluau était, semble-t-il, un mulâtre des Indes occidentales au service de la Compagnie de Monts à titre d'interprète..$^{15}$ Les mulâtres étaient souvent employés par les Européens qui trafiquaient avec les Indiens de la Nouvelle-Angleterre et de la Virginie et on leur accordait de vrais salaires et certains avantages marginaux. Les Hollandais qui pratiquaient le troc sur la rivière Hudson dans les années 1613-1614, avaient aussi à leur emploi un mulâtre, Jan Rodriguez. ${ }^{16}$ Il existe un document indiquant

\footnotetext{
14 Archives des Etats généraux, La Haye, Archives des Etats généraux, no $554,83 \mathrm{v}-84$.

15 Ch. Beaurepaire, La Compagnie de Monts, 4-11. - Archives nationales de France, registre de Cuvillier, XV, 19, f. 945.

16 AVA, Archives notariales, no 197, f. $614 \mathrm{v}-615$.
} 
que Berrewijns et ses associés se préparaient dès 1607 à retourner au Canada. Ils estimaient pouvoir utiliser les services de $\mathrm{Da}$ Costa pour leur commerce avec les Indiens du Canada. ${ }^{17}$ Lorsque Ralluau revint de Hollande en 1607 après s'être occupé de l'affaire des biens volés à la Compagnie de Monts, Mathieu Da Costa "brûla la politesse" à son patron français pour s'engager aux Hollandais. ${ }^{18}$ A la suite de cette désertion, Ralluau revint en toute hâte en Hollande. Il y dépensa beaucoup de temps et d'argent en procès devant les cours hollandaises. ${ }^{19} \mathrm{En}$ 1607 les notaires d'Amsterdam furent accaparés par toutes les parties impliquées dans l'affaire du Lion Blanc. Il se peut que la Compagnie Berrewijns ait été mise au courant par ses amis français, de l'action imminente de de Monts à la cour de France. On voit par plusieurs actes passés devant le notaire d'Amsterdam, Frans Bruyningh que la Compagnie prit soin d'étayer sa version des événements de 1606. Il est déclaré dans ces actes que le capitaine Lonck avait fait des prises légales en s'emparant d'un navire espagnol et d'un navire portugais et avait indiqué leur cargaison. Mais il n'était pas fait mention des navires de la Compagnie de Monts ni d'un commerce quelconque à Tadoussac. ${ }^{20}$ La lecture de ces documents, nettement rédigés en vue d'embrouiller n'importe lequel investigateur, porterait à conclure que la Compagnie n'avait jamais mis le pied sur le sol canadien. Bien plus, il y avait une importante raison de faire rédiger des documents légaux par un notaire de la ville d'Amsterdam.

Apparemment, Nicolas de Bancquemaire ne se fiait pas à ses associés hollandais. Français, il était illégal pour lui d'aider des étrangers à commercer dans des territoires protégés par une charte royale. Il se peut qu'il ait soupçonné ses associés hollandais de chercher à le tromper à la première occasion qui se présenterait. C'est peut-être pour cette raison qu'une déclaration fut rédigée pour légaliser l'arrangement définitif pardevant le notaire Bruyningh, le 11 décembre 1606. On fit une estimation de la valeur de la marchandise, et celle-ci fut alors transportée ailleurs. Cette décision fut prise, soit parce que cette marchandise spéciale pouvait rapporter beaucoup plus

17 AVA, Archives notariales, no 105, f. 198-199.

18 Voir note 15.

19 Beaurepaire fait mention d'un contrat fait à Amsterdam, au sujet du Noir Da Costa. On n'a pu retracer ce contrat malgré tous les efforts et on présume qu'il a été perdu. Ce fut probablement le cas de plusieurs documents qu'il mentionne comme ayant existé au tabellionage de Rouen.

20 AVA, Archives notariales, no 106 , f. $54 \mathrm{v}-55$; no 195, f. $85 \mathrm{v}-86 \mathrm{v}$; no 105, f. 198-199. 
ailleurs, soit parce que les marchands voulaient empêcher la Compagnie de Monts de recouvrer les fourrures et les autres marchandises volées. De Bancquemaire en reçut sa part. Selon toute apparence l'expédition de 1606 fut financièrement un grand succès. ${ }^{21}$ Le voyage de Ralluau dans l'intérêt de de Monts fut par ailleurs un échec.

Quand le secrétaire de de Monts arriva en Hollande, il avait deux affaires à régler, ce qui l'obligea à faire la navette entre Amsterdam et Rotterdam. Ce n'est pas que la distance entre ces villes soit considérable car elle dépasse à peine soixante-dix milles, mais à l'époque une grande étendue des deux provinces hollandaises n'avait pas encore été asséchée. Au prix de dépenses considérables et après une longue correspondance, Ralluau obtint quatre canons et deux ancres, mais ces pièces n'avaient pas appartenu au navire de la Compagnie de Monts. Elles provenaient plutôt d'un navire espagnol et étaient de piètre qualité. ${ }^{22}$ Ralluau les vendit à Louis Delbecque, l'un des associés de la Compagnie Berrewijns. ${ }^{23}$ Il recut la moitié du prix de la vente - 245 francs et 14 sous - à Amsterdam et, le 23 avril 1607, Jacob Bolle, neveu de Delbecque et marchand d'Amsterdam, lui versa l'autre moitié à Rouen. ${ }^{24}$ Les frais occasionnés par ces démarches et ces déplacements représentaient sans doute plusieurs fois la valeur des canons et des ancres, ce qui prouverait que de Monts et Ralluau étaient des hommes d'affaires médiocres. Du moins c'était l'opinion des Hollandais, parce que plusieurs années plus tard, ils déclaraient ouvertement "De Griekenverstaan de negotie niet", ce qui signifie "Les Grecs (les Français) ne s'y entendent guère en affaires". ${ }^{25}$ En conséquence, il semble bizarre que Champlain ait confié à Ralluau en 1615 la tâche de poursuivre en justice la firme Georges et Macain de La Rochelle. ${ }^{26}$ L'affaire de Da Costa, le "nègre évadé", semble avoir été considérée comme ayant une importance beaucoup plus grande.

On ne connaît pas avec certitude le motif qui poussait de Monts à attacher tant d'importance à Da Costa. Cet homme

21 S. Hart, Prehistory of the New Netherland Company, 14.

22 Ibid., 15.

23 Delbecque était le grand-père de Pieter Carpentier, un des grands gouverneurs des Indes orientales dans les années 1620. Archives de la ville de Dordrecht, Archives notariales, no 11, f. 615v.

24 Archives de la Seine-Maritime, Tabellionage de Rouen (meubles), $1^{\text {ère }}$ série, $1^{\text {er }}$ janvier au 30 juin 1607.

25 Archives de la Loire-Inférieure, C., no 652.

26 Archives de la Seine-Maritime, Tabellionage de Rouen (meubles). 
avait probablement une grande valeur aux yeux de ceux qui préparaient l'expédition de 1608. En 1607, un projet avait été formé en vue d'envoyer Champlain en Nouvelle-France. Ce dernier avait pour mission d'établir une habitation permanente à Québec et d'explorer le pays des Iroquois. L'expérience des Hollandais avait prouvé que les mulâtres étaient peu dignes de confiance, et, à l'exemple des Français, ils commencèrent bientôt à former leurs propres hommes au métier d'interprète et d'agent de liaison auprès des Indiens. ${ }^{27} \mathrm{Si}$ de Monts s'était proposé d'avoir recours aux services de Da Costa en 1608, il fut certainement déçu, car le mulâtre ne fut renvoyé à Rouen qu'au printemps de 1609.28 On ignore si les Hollandais utilisèrent les services de Da Costa; toutefois, le retour de ce dernier se révéla important pour Nicolas de Bancquemaire.

C'est après beaucoup de difficultés que l'on parvint à faire comparaître Da Costa devant les tribunaux de Hollande. Apparemment, le mulâtre n'avait jamais été esclave. Par conséquent, Ralluau se vit obligé de demander l'aide de Nicolas de Bancquemaire, l'associé en affaires de "Berrewijns et associés". De Bancquemaire était tout disposé à collaborer avec Ralluau, mais à condition qu'il puisse retourner en France sans crainte d'être traduit en justice par de Monts pour son rôle dans l'affaire de Tadoussac en 1606. De toute façon, il semble que de Bancquemaire ait pris son temps à "collaborer", à tel point que Ralluau dut faire un long séjour en Hollande, ce qui lui occasionna des frais considérables. Ce n'est qu'en 1609 que Da Costa fut remis entre les mains de Ralluau dans la ville de Rouen. ${ }^{29}$ Cependant la libération du mulâtre ne signifiait pas que de Bancquemaire était au bout de ses peines.

Quand Nicolas revint en France, de Monts n'était pas au courant de la promesse faite par Ralluau, ou, du moins, il fit mine de n'en rien savoir. De Bancquemaire tenta vainement de recouvrer les dépenses occasionnées par les démarches qu'il avait entreprises en Hollande pour le compte de de Monts. Bien plus, ce dernier le traduisit en justice pour les pertes que lui-même avait subies. Ces divers procès traînèrent devant les tribunaux français jusqu'au 8 janvier 1619. De Bancquemaire dut verser la somme de 1,000 livres à Ralluau, mais il semble que de Monts n'ait rien reçu. En 1619, de Monts et Nicolas

27 AVA, Archives notariales, no 197 , f. $614 \mathrm{v}-615$; no 198 , f. 97-98.

28 Archives nationales de France, registre de Cuvillier, XV, 19, f. 945.

29 Ch. Beaurepaire, La Compagnie de Monts, 5-10. - Registre de Cuvillier, ibid. 
de Bancquemaire étaient tous deux au service de la France, le premier comme gouverneur de la ville de Poitiers, et le second en qualité de Capitaine de la Marine du Ponant et fermier des Aides.30 Abstraction faite de l'avenir qui s'ouvrait devant de Monts, il est certain qu'en 1607 ni ce dernier ni sa Compagnie n'étaient en mesure de contribuer à l'essor de la NouvelleFrance. En 1608, afin de l'indemniser de ses pertes, on octroya au sieur de Monts le monopole de la traite des fourrures pour une période d'une année. Par la suite, de Monts joua un rôle de plus en plus effacé dans l'histoire de la Nouvelle-France.

Pour bien comprendre les activités auxquelles se livraient les Hollandais en 1606, il faut connaître un peu les antécédents de ceux qui y prirent part, ainsi que l'état des relations commerciales de la France au début du XVII ${ }^{e}$ siècle. Il ne faut pas oublier que les premiers efforts véritables de la France pour coloniser le Canada furent déployés au moment où le commerce de la république hollandaise connaissait une expansion remarquable. En quelques décennies, cet essor allait donner à ce pays la suprématie mondiale du commerce du transport. En réalité, les efforts du peuple hollandais en vue d'étendre leur commerce étaient, en partie, dirigés contre la France. La position particulière des Huguenots dans les domaines politique et économique, position assurée par l'Edit de Nantes, favorisait les marchands protestants de nationalité hollandaise qui désiraient établir des marchés à l'ouest et au sud de la France. Le gouvernement français tenta d'endiguer le flot des négociants et des artisans hollandais et wallons en décrétant que les marchands et autres hommes d'affaires étrangers devaient obtenir la citoyenneté française avant d'exercer leur activité dans certaines régions et certaines villes. Cependant, cette barrière se révéla peu efficace et l'influence commerciale des Hollandais en France continua de s'accroître. ${ }^{31}$

En fait, l'infiltration des Hollandais dans le monde des affaires en France prit de telles proportions que, même en 1758, on se plaignait encore que le prix des produits français dans les colonies était fixé par les commerçants de la Hollande.32 Peut-être exagère-t-on quelque peu, mais ceux qui étudient

$30 \mathrm{Ch}$. Beaurepaire, La Compagnie de Monts, 10-11.

31 G.-Ch. Fagniez, L'Economie sociale de France sous Henri IV (Paris, 1897), 26ss, 85-87-192ss. - G. Martin, La grande Industrie sous le règne de Louis XIV (Paris, 1898), chapitres V-VI. Des études plus récentes se trouvent dans les travaux et articles de H. Sée et M. Morineau.

32 Thomas Pichon, Lettres et Memoires pour servir à l'histoire naturelle, civile et politique du Cap Breton (La Haye, 1760), 168-173. 
l'histoire économique de la France sont d'avis que l'agressivité des trafiquants hollandais, l'activité de la bourse d'Amsterdam et la puissance de la marine marchande de Hollande ont eu sur la vie commerciale de la France une influence comparable à celle que le régime économique des Etats-Unis exerce actuellement sur l'Europe occidentale. En admettant que les fourrures de la Nouvelle-France comptaient peu en regard des produits venant de l'Orient, on ne peut nier que le commerce des pelleteries joua un rôle important dans l'expansion primitive des colonies de l'Amérique du Nord, en particulier en NouvelleFrance. ${ }^{33}$ En outre, un faible investissement, surtout dans les premiers temps du commerce des fourrures, pouvait rapporter des bénéfices considérables. En conséquence, il était facile de prévoir que les Hollandais se rendraient vite compte des possibilités qu'offrait ce genre de négoce. Cependant, les marchands de fourrures de la république hollandaise avaient des raisons particulières de s'intéresser aux tentatives de la France qui voulait exploiter une colonie dont la vie économique serait assurée par les revenus provenant de la traite des pelleteries.

En 1608, les Hollandais étaient sur le point d'accaparer tout le marché de la fourrure en Europe, surtout en raison d'un traité commercial des plus avantageux qu'ils avaient conclu avec la Russie. ${ }^{34}$ Toutefois, au début du XVII ${ }^{e}$ siècle, le quasimonopole de la Hollande se trouva vite menacé par les stocks de fourrures de plus en plus considérables qui arrivaient à Rouen en provenance de l'Amérique. ${ }^{35}$ Les traiteurs se procuraient ces fourrures à des prix très bas chez les tribus primitives et, d'après certains marchands hollandais, les peaux étaient d'une qualité supérieure à celles qui venaient d'Archangel. ${ }^{36}$ C'est ce qui explique qu'à cette époque plusieurs négociants de la Hollande vinrent s'établir à Rouen, à La Rochelle, à Bayonne et dans d'autres villes françaises de la côte de l'Atlantique,

83 Th. Funck Brentano, éd., Antoine de Montchrétien, Traicté de l'Oeconomie politique (Paris, 1889). - Jean Eon, Le Commerce Honorable (Nantes, 1646). - P. Bonassieux, Les Grandes Compagnies de Commerce (Paris, 1892). - H. Pigeonneau, Histoire du Commerce de la France (Paris, 1889), vol. I. - H. Sée, Franzósjsche Wirtsschafts Geschichto (Jena, 1930), vol. I. - H. A. Innis, The Fur Trade in Canada (Toronto, 1956).

34 P. Chrisler Phillips, The Fur Trade (Norman, Oklahoma, 1961), I: 146-147. Voir aussi la note 3 .

$35 \mathrm{H}$. P. Biggar, The Early Trading Companies of New France (Toronto, 1901), 1-62.

36 J. F. Jameson, Narratives of New Netherland 1609-1664 (New York, 1909), 76-77. 
villes qui depuis un certain temps entretenaient des relations suivies avec les "terres à fourrures" de l'Amérique du Nord. Souvent, il est vrai, d'autres raisons jouèrent un rôle décisif dans l'établissement de colonies hollandaises dans les ports de France. Par exemple, le fait que les pêcheries de la Baltique avaient un besoin constant de sel décida un certain nombre de marchands hollandais à se fixer à Brouage, ville natale de Champlain, ainsi que dans d'autres centres de l'industrie salicole de la France. D'autres aussi s'intéressaient au commerce du transport côtier de la France qui n'avait cessé de péricliter depuis 1580 et que la marine marchande hollandaise était alors en train d'accaparer. Toutefois, il importe peut-être de souligner que tous les marchands hollandais qui, dix ans plus tard, allaient prendre une part active au commerce des fourrures, sur l'Hudson et la Delaware, s'installaient à cette époque dans les ports français de l'Atlantique ou y déléguaient des parents ou autres représentants. ${ }^{37}$ L'une de ces familles de négociants était les van Liebergen.

Les van Liebergen se fixèrent à Rouen au début du XVII siècle. Parmi ceux avec qui ils étaient en relations d'affaires, il y avait Jacques, Nicolas et Nathaniel de Bancquemaire, ${ }^{38}$ qui participaient activement à la traite des fourrures en NouvelleFrance. ${ }^{39}$ Arnout van Liebergen, un des actionnaires de la Compagnie hollandaise des Indes orientales, fut l'un des premiers à trafiquer sur la rivière Hudson. En 1614, il devint l'un des fondateurs de la Compagnie de la Nouvelle-Hollande. Les membres de la branche française des Liebergen étaient des amis intimes de la famille hollandaise Dircksz (Dericq). Celle-ci, en plus de faire le commerce des fourrures et de fabriquer des chapeaux, possédait de gros intérêts dans le commerce des pelleteries au Canada. Leur fils, Nicolas Dericq, qui prit la succession du commerce, fit un second mariage en 1640 et épousa Marie de Caen, veuve de Raymond de la Ralde. ${ }^{40}$ L'un

${ }^{37}$ AVA, Archives notariales. Voir la série de contrats d'affrètement par ordre de villes de destination, v.g., Brouage, Saint-Malo, etc. P. Bonnassieux, Les grandes Compagnies, 245-259. - Z. W. Sneller et W. S. Unger, Sources for the History of the Trade with France (La Haye, 1930), 660-662.

38 AVA, Archives notariales, no 122 , f. $181 \mathrm{v}$; no 352 , f. $2 \mathrm{v}$; no 692 , f. $32 \mathrm{v}, 36 \mathrm{v}$; no 125 , f. $232,236 \mathrm{v}$, 139 ; no 210 , f. $110 \mathrm{v}$; no 136 , f. $177 \mathrm{v}-178$.

39 Bibliothèque Nationale de France. Interrogatoire de Pierre de Gua; 2 avril $1612,8,8^{\circ} \mathrm{Z}$, no 11535 . - J. Felix, éd., Comptes rendus des Echevins de Rouen (Rouen, 1890), I, passim.

40 AVA, Archives notariales, no 197 , f. $614 \mathrm{v}-615 \mathrm{v}$; no 136, f. $177 \mathrm{v}-178$.

- J. G. van Dillen, The oldest list of shareholders of the East India 
des capitaines au service de la firme van Liebergen était Thijs Volckertsz Mossel. ${ }^{41}$

Mossel figure dans l'Histoire de la Nouvelle-France de Lescarbot sous le nom de capitaine Foulques (Volckertsz).42 Se trouvant aux Antilles en 1597 et 1598, il explore la côte de l'Atlantique depuis les îles antillaises jusqu'à l'Orénoque et trafique avec les indigènes qu'il paie avec des colliers de verroterie et autres brimborions. En 1613, il fait du commerce sur l'île Manhattan ou dans les régions avoisinantes pour le compte des Hollandais Hans Claesz, van Liebergen et autres négociants. Plus tard, il se met au service de marchands indépendants de la Compagnie Hendrick Eelckens qui font du commerce sur les rivières Hudson et Delaware, de 1618 à 1623. Le subricargue de Mossel est Hans Jorisz Hontom qui devint plus tard commis et commandant de Fort Orange. ${ }^{43}$

Un autre Néerlandais, Lambert van Tweenhuyzen, le dernier directeur de la Compagnie de la Nouvelle-Hollande ne se fixa jamais en France, mais entretint des relations commerciales étroites avec la firme Georges et Macain de La Rochelle. Louis Hébert, qui prit part à l'expédition de Port-Royal avec la Compagnie de Monts et qui fut le premier colon de Québec en 1617, était un cousin de ces négociants français. ${ }^{44}$ Les deux compagnies précitées s'intéressaient aux pêches à la baleine de Spitsbergen et recrutaient leurs harponneurs dans les localités basques du golfe de Gascogne. D'ailleurs, c'est là que Rotterdam embauchait la plupart des équipages de ses baleinières. Les activités commerciales de van Tweenhuyzen s'étendaient d'Archangel à l'Afrique du Nord et à Constantinople, ${ }^{45}$ et ce sont probablement les

Company, Amsterdam Chamber (La Haye, 1959), 151. Un des actionnaires était Jacques de Caen. S. Hart, Prehistory of the New Netherland Company, 11ss. - "Nicolas Dericq, grand marchand de Rouen au XVIIe siècle", Bulletin de la Commission des Eglises wallonnes (La Haye, 1896), 129-130. 41 S. Hart, Prehistory New Netherland Company, 21-32, 35n, 38, 51, $56-60,68,74,75,77-94,97$. 290-301.

42 W. L. Grant, ed., Lescarbot, Histoire de la Nouvelle-France, II:

43 S. Hart, Prehistory of the New Netherland Company, 589, 77ss. A. F. J. van Laer, Van Rensselaer Bowier MSS (Albany, 1908), 302-304. $44 \mathrm{M}$. Bouvel, "L'Apothicaire Louis Hébert", Revwe de l'histoire de la Pharmacie (Paris, 1954), no 143, 9.

$45 \mathrm{~S}$. Hart, "The first whaling journeys of the Netherlanders", Amstelodanum (Amsterdam, 1957), 27-64. Contient aussi une biographie de van Tweenhuyzen. - Archives de la ville de Rotterdam, Archives notariales, e.g., Protocal Vogel, VII : 25; VIII: 46; Prot. Kieboom, 12 sept. 1633; Prot. Heusen, 74 (1617). Voir aussi Bijdragen Historisch Genoot- 
rapports que ce dernier entretenait avec la firme Georges et Macain qui l'incitèrent à faire le commerce des fourrures avec la Compagnie de la Nouvelle-Hollande. Ceux qui étudient l'histoire des premiers temps de la colonie connaissent bien cette société commerciale de La Rochelle, parce que celle-ci avait un intérêt dans la Compagnie de Monts, et qu'elle participa plus tard aux entreprises de Poutrincourt, de Biencourt et d'autres armateurs. D'ailleurs, on retrouve souvent les noms de Georges et Macain dans les ouvrages de Lescarbot et de Champlain.46 Toutefois, le négociant hollandais qui exerça la plus forte influence sur le marché de la fourrure à cette époque, fut probablement Arnout Vogels.

Vogels était apparenté aux Eelckens et aux Duysterloo, et tous étaient alliés par mariage à la puissante société commerciale des Jabach. Ces derniers avaient d'ailleurs été surnommés les "Fuggers de l'Ouest". Les Duysterloo jouèrent un rôle important dans le commerce français des fourrures et ils paraissent avoir été les maîtres de ce négoce au cours des années 1630 . Les relations que Vogels entretenait avec Rouen avaient trait au commerce des fourrures. En 1610, Vogels envoie un de ses capitaines aux Antilles et dans "les pays avoisinants" avec mission d'effectuer une reconnaissance dans les "terres à fourrures" qu'Henry Hudson venait de découvrir. ${ }^{47}$ L'année suivante, il s'associe à Lodewijck Vermeulen et Jehan Andries (Andrieu), deux citoyens de la ville de Rouen, afin de faire du commerce au Canada. ${ }^{48}$ Leur subrécargue se nommait François DupontGravé et était l'un des associés de de Monts et Champlain. Dans ses écrits, ce dernier mentionne Lodewijck Vermeulen et Mathijs Duysterloo comme directeurs de la Compagnie qui porte son nom. ${ }^{49}$ Il est probable que l'entreprise mise sur pied par

8chap (Utrecht, 1898), 365ss. En 1617, 150 pêcheurs étaient à l'emploi de la Compagnie hollandaise du Nord.

40 E. Trocmé et M. Delafosse, Le Commerce rochellais de la fin du $X V I^{e}$ au début du XVII e siècle (Paris, 1956), 122-123, 136, 150-152, 167-168. - S. Hart, Prehistory of the New Netherland Company, 40. - W. L. Grant, ed., Lescarbot, e.g., 268-274. - H. P. Biggar, ed., The Works of Samuel de Champlain (Toronto, 1922), e.g., I: 228.

47 Peter Paul Trippen, Jabach, die Fugger Familie des Westen (Cologne, 1938), généalogie. - Bibliothèque Nationale de France, Factum pour Nicolas Libert, $4^{\circ} \mathrm{Fm}$, no 19503. - Archives nationales de France, Minutes de Dournel, XXXV, nos 75, 151. - S. Hart, Prehistory of the New Netherland Company, 42. - R. LeBlant et R. Baudry, Nouveaux Documents sur Champlain et son époque (Ottawa, 1967), 151, 181, 182, 194, $200,203,212,219,225,295,348,388,403,418,419 \mathrm{n}, 466,468,418 \mathrm{n}$ (Bellois).

${ }_{48 \mathrm{AVA}}$ Archives notariales, no 125, f. 105-106; no 269, f. $202 \mathrm{v}-203$.

49 H. P. Biggar, Champlain's Works, IV: 355-356. 
Vogels sut profiter de l'occasion qu'avait fait naître la période de libre concurrence qui dura de 1609 à 1613 dans la vallée du Saint-Laurent. En 1613, Vogels s'intéresse au commerce des fourrures de la Nouvelle-Hollande et y fait des affaires jusqu'à sa mort en 1620.50 Un autre négociant en vue de la Hollande fut Hans Hunger.

Hans Hunger, marchand d'épices et gros actionnaire de la Compagnie hollandaise des Indes orientales, s'intéressait aussi au commerce français des fourrures. En 1606, ses employeurs envoyèrent Lonck au Canada faire un raid contre la Compagnie de Monts. A titre de directeur de la Chambre d'Amsterdam de la Compagnie des Indes orientales, Hunger fut sans doute le premier à entendre parler des "terres à fourrures" de la rivière Hudson découvertes par Henry Hudson en 1609. Il devint directeur de la Compagnie van Tweenhuyzen, connue plus tard sous le nom de la Compagnie de la Nouvelle-Hollande et qui trafiqua sur les rives de l'Hudson et de la Delaware. ${ }^{51}$ Un autre personnage digne d'intérêt est Jacobsz Eelckens.

Eelckens était l'aîné d'une famille qui s'était établie à Rouen en l'an 1600 et qui avait entretenu des rapports étroits avec les Eelckens d'Amsterdam. Son fils, le jeune Jacob, fut élevé à Rouen où il s'initia au commerce des fourrures. Il travailla en qualité de subrécargue pour la Compagnie Vogels et van Tweenhuyzen, puis se joignit à son oncle, un commerçant indépendant de la Compagnie de la Nouvelle-Hollande pendant la période de 1618 à 1623. Les Eelckens occupèrent dans le commerce hollandais des fourrures la même place que Nicolas de Bancquemaire dans le commerce français. ${ }^{52}$ D'autres négociants hollandais eurent des relations d'affaires avec le monde de la fourrure en France, mais leur importance est secondaire.

Les Berrewijns, les Timmermans et les Delbecque étaient tous des marchands hollandais très en vue qui avaient des intérêts dans le commerce français. Leurs noms reviennent régulièrement dans les actes notariés d'Amsterdam, de Rotterdam et de Dordrecht. Cependant, abstraction faite de l'opération de 1606, ces négociants ne semblent pas s'être intéressés au Canada si l'on excepte une expédition isolée en vue de réaliser

50 Voir la note 47. R. Le Blant et M. Delafosse, "Les Rochelais dans la Vallée du Saint-Laurent, 1599-1618", Revue d'histoire de l'Amérique française (Montréal, 1956), X: 338. - S. Hart, Prehistory of the New Netherland Company, 43-44.

51 S. Hart, Prehistory of the New Netherland Company, 47-48. AVA, Archives notariales, no 106, f. 54v-55.

52 S. Hart, ibid., 54-55. 
des gains faciles. Peut-être furent-ils rebutés par l'attitude qu'adoptèrent les Etats généraux de la Hollande en 1607 vis-à-vis du commerce au Canada. Il est vrai qu'ils projetaient de faire un second voyage et qu'ils demandèrent d'être indemnisés quand le gouvernement hollandais fit échec à leurs desseins. Ces marchands s'intéressaient surtout aux produits des pays orientaux, aux céréales et aux pêcheries. ${ }^{53}$ Toutefois, de même que les trafiquants de fourrures de la Hollande, ils firent sentir leur présence dans les entreprises françaises en Nouvelle-France.

Les personnes dont nous venons de parler ne représentent qu'une poignée des négociants hollandais qui s'étaient fixés en France ou qui faisaient du commerce dans ce pays par l'intermédiaire d'associés ou de relations d'affaires. Les archivistes d'Amsterdam et de La Rochelle ont rassemblé de nombreux documents notariés qui prouvent hors de tout doute que, dès le début, les commerçants de la Hollande étaient bien au courant de la menace constituée par l'arrivée des fourrures de l'Amérique du Nord sur le marché euronéen ${ }^{64}$ Aussi, la Compagnie de Monts ayant été la première entreprise à se livrer à une exploitation bien organisée des "terres à fourrures" de la Nouvelle-France, il n'est pas étonnant qu'elle ait été la cible d'une opposition énergique de la part des marchands hollandais. Cette opposition se traduisait par des raids effectués par des navires puissamment armés au cours desquels on s'emparait des marchandises et autres biens. Quand les compagnies de moindre importance se fusionnèrent pour former les grandes sociétés commerciales du XVII ${ }^{e}$ siècle, les raids continuèrent, mais avec beaucoup plus d'envergure. ${ }^{55}$ Le fait que de Monts détenait un monopole rendait sa situation encore plus difficile, parce qu'en plus d'être en butte aux incursions des étrangers, il était la cible des attaques de ses propres concitoyens exclus du commerce des fourrures.

La Compagnie de Monts aurait sans doute été incapable de se maintenir bien longtemps face à l'hostilité de la plupart des marchands de la côte française de l'Atlantique. En plus d'effec-

${ }^{53}$ AVA, Archives notariales et contrats d'expédition. Voir l'index des marchands. - S. Hart, Prehistory of the New Netherland Company, $15,50 \mathrm{n}$.

54 AVA, Archives notariales et contrats d'expédition - Archives de la Charente-Maritime, Tabellions. Cette concurrence devint de la coopération. A la fin du 17 e siècle c'est le commerce hollandais qui absorba la plus grande partie du surplus de fourrures de la Nouvelle-France. Voir la communication de M. Delafosse au Colloque international de l'histoire coloniale, Ottawa, novembre 1969.

1965), passim. 
tuer des raids contre la Compagnie, ces marchands exerçaient de fortes pressions politiques sur les dirigeants de la Bretagne et de la Normandie. A leur tour, ces provinces affichaient leur répugnance à collaborer avec le gouvernement français, soit en refusant de signer la charte octroyée à la Compagnie de Monts, soit en montrant bien peu d'empressement à apposer leur signature. ${ }^{56}$ Les Basques constituaient pour de Monts un danger encore plus grand, parce qu'ils échappaient à l'autorité de la France et que, par conséquent, seule la force pouvait leur faire entendre raison. La France se trouvait dans l'impossibilité de leur imposer sa volonté par les armes et les événements qui allaient suivre le démontrèrent sans l'ombre d'un doute. ${ }^{57}$ De Sully, premier ministre du roi Henri IV, ne prisait guère les entreprises coloniales. Quant aux chapeliers de Paris, ils estimaient que les monopoles nuisaient à leurs intérêts parce qu'à cause d'eux les peaux de castor se vendaient plus cher. ${ }^{58}$ Le seul appui sur lequel la Compagnie de Monts pouvait compter était la cour du roi de France. Toutefois, Henri IV abandonna de Monts en 1608, quand la pression exercée par les Etats généraux de la Hollande mit fin à ses ambitions commerciales.

La charte originale de la Compagnie de Monts rédigée en 1604 stipulait que seuls les Français pouvaient participer à l'œuvre entreprise en Nouvelle-France. Rien n'autorise à penser que cette décision visait les Hollandais, mais le fait qu'Henri de Navarre avait organisé en 1604 une société commerciale sur le modèle des compagnies anglaises et hollandaises des Indes orientales, n'est pas sans signification. ${ }^{59}$ Cependant, alors que ces deux dernières étaient le résultat du fusionnement de petites compagnies, fusionnement qui visait à éliminer la concurrence ruineuse et à conjuguer les énergies et les capitaux afin d'accrô̂tre les moyens d'action, le commerce français, lui, ne cessait de se détériorer sous l'effet des restrictions de plus en plus nom-

${ }^{56} \mathrm{Ch}$. et $\mathrm{P}$. Bréard, Documents relatifs à la Marine normande (Rouen, 1889), 92ss. - H. Michelant et P. Ramé, Relations originales du voyage de Jacques Cartier au Canada en 1534 (Paris, 1867), 21-30.

${ }^{57}$ H. P. Biggar, ed., Works of Samuel de Champlain, I: 230-239. Voir aussi la note 44 .

58 Archives nationales de France, Série E, 14s, f. 71.

59 G. Fagniez, L'économie sociale, 26ss, 85-86, 193ss, 142-143, 157. Les transactions des Hollandais, spécialement celles de Le Maire et de Lyntgens sont rapportées en détail d'après les sources originales, dans J. G. van Dillen, "Isaac Le Maire et le commerce des actions de la Compagnie des Indes occidentales", Economisch Historisch Jaarboek (La Haye, 1930), XVI: 1-228. - Collection de Documents relatifs à l'histoire de la Nouvelle France (Québec, 1883), I: 40-49. 
breuses imposées par le gouvernement et à la suite de l'infiltration sans cesse croissante des marchands anglais et hollandais.80 En 1604, Henri IV se vit contraint de demander à certains mécontents parmi les négociants hollandais de l'aider à mettre sur pied un organisme pour sa Compagnie des Indes orientales.1 Dès 1607, les Etats généraux de la Hollande avaient démontré qu'ils ne toléreraient aucune ingérence, même de la part de ses alliés, dans le commerce hollandais. Aussi, quand en 1608, le roi de France chercha à attirer des Hollandais afin que sa compagnie puisse profiter de leur expérience, de leurs navires et de leur personnel, le gouvernement hollandais lui fit savoir en termes non équivoques qu'il opposerait les mesures les plus énergiques à de telles tentatives. En 1609, le gouvernement français expliqua aux Etats généraux que la charte octroyée à de Monts n'avait jamais eu pour but d'empêcher les Hollandais de faire du commerce en Nouvelle-France. ${ }^{62}$ La deuxième compagnie à jouir d'un monopole, la Compagnie de Champlain, avait comme directeurs deux négociants hollandais, Lodewijck Vermeulen et Mĩathijs Duysterioo. ${ }^{\hat{\sigma} \overline{3}}$

Bien que l'expédition hollandaise de 1606 ne fût pas le motif principal de la dissolution de la Compagnie de Monts, il est permis de conclure que c'est la goutte d'eau qui fit déborder le vase. Le raid eut aussi certains effets secondaires. Ainsi, quand de Monts porta sa cause devant les Etats généraux et l'Amirauté d'Amsterdam, il attira, par le fait même, l'attention des marchands hollandais sur le commerce des fourrures au Canada. La réaction de ces derniers contre ce qui était, selon eux, un empêchement à la libre concurrence, influença le gouvernement de la Hollande et explique la position hollandaise adoptée en 1609 au sujet du monopole exercé par la France au Canada. C'est peut-être là l'une des raisons qui incita Henri IV à décider que la liberté de commerce au Canada serait dans l'intérêt de la France. Enfin, il est permis de conclure que le succès de la première intervention hollandaise dans le commerce des fourrures au Canada ne pouvait qu'encourager les trafiquants de la Hollande à continuer leurs efforts en vue de s'assurer une part du marché de la fourrure en Amérique.

60 P. Bonnassieux, Les grandes Compagnies, 245-259. - Z.W. Sneller et W. S. Unger, Sources for the History of the Trade with France, 660-662. 61 P. Bonnassieux, Les grandes Compagnies, 245-259.

62 C.-B. Petitot, éd., Négociations du Président Jeannin (Paris, 18191829), XV: 232. - M. Berger de Xivrey, éd., Lettres missives de Henri IV, VII: 897-899. - Archives publiques du Canada, C11 A, Amérique, 11. 63 H. P. Biggar, Works of Samuel de Champiain, IV : 356-357. 